\title{
Development and validation of a diet quality index for older adults
}

\author{
N. Dorrington ${ }^{1}$, R. Fallaize ${ }^{1,2}$, M. Weech ${ }^{1}$ and J.A. Lovegrove ${ }^{1}$ \\ ${ }^{1}$ Hugh Sinclair Unit of Human Nutrition, University of Reading, Reading, RG6 $6 \mathrm{AH}$ and \\ ${ }^{2}$ School of Life and Medical Sciences, University of Hertfordshire, Hatfield, AL10 9AB.
}

UK life expectancy has risen ${ }^{(1)}$, yet this has been accompanied by a longer time lived with morbidity, impacting healthcare costs and quality of life. Appropriate dietary choices are fundamental in later life. However, few well-evidenced nutritional recommendations exist for older adults, challenging provision of personalised nutrition. Diet quality indexes (DQIs) are useful measures of nutritional adequacy, but component selection can limit their validity in older adults. Therefore, this research aimed to propose nutritional recommendations for older adults (aged $\geq 65 \mathrm{y}$ ) and tested three variations of a DQI used for assessing adherence.

The literature was systematically reviewed to propose recommendations for all selected nutrients. Current UK guidelines were retained if evidence-based and age-specific, else Web of Science was searched up to September 2017. A standard protocol ${ }^{(2)}$ was used to develop the DQIs: a food-group based DQI (FBDQI), a food and nutrient based DQI (FNBDQI) and a healthy lifestyle DQI (HLDQI). Decisions regarding components, criteria and scoring were guided by the literature search and nutritional recommendations. The DQIs were validated using data for adults $\geq 65 \mathrm{y}$ from years 2-6 of the National Diet and Nutrition Survey rolling programme $(n=873)^{(3)}$. Associations between calculated scores and nutrient intake, health status measures and metabolic markers were statistically analysed $(\mathrm{P}<0.05$ was significant).

From a total of 64,708 identified papers, 202 studies guided setting of the nutritional recommendations. Evidence supported changes to current population protein $(1.2 \mathrm{~g} / \mathrm{kg}$ body weight/day), calcium $(1000 \mathrm{mg} / \mathrm{day})$, folate $(400 \mu \mathrm{g} / \mathrm{day})$, vitamin B12 $(2.4 \mu \mathrm{g} /$ day) and fluid recommendations (1.6L/day women, 2L/day men) for those $\geq 65 \mathrm{y}$. The FBDQI and FNBDQI had 12 equally weighted components (vegetables, fruit, lean protein, oily fish, low fat dairy, wholegrain carbohydrates, free sugars, sodium, fat, fluid, alcohol, physical activity). Quantification was based on the EatWell Guide $e^{(4)}$ or numbers of standard portions required to meet certain nutrient intakes for food-group components, and was directly from the recommendations for nutrient components. Graduate scoring was implemented, with values adopted relative to the extent to which component recommendations were met. Scores were summed to a maximum of 120 , where higher scores indicated greater adherence. The HLDQI was a modified version of the FNBDQI, including a smoking component. Higher DQI scores were significantly associated with increased odds of meeting almost all nutritional recommendations, lower obesity, medication use, systolic blood pressure (FNBDQI and HLDQI), serum fasted triglyceride, C-reactive protein, fasting glucose, odds of poor self-assessed health and hypertension, and higher high-density lipoprotein cholesterol.

The results indicate the DQIs to be valid methods of assessing nutritional adequacy in older adults, and that adherence to the proposed nutritional recommendations and dietary pattern may help reduce risk of disability and disease. Quantifying certain components as nutrients enhanced the efficacy of the FNBDQI, but benefits of measuring smoking were minimal. Investigations into associations with other indicators of morbidity and mortality are needed to further evaluate its efficacy as a measure of dietary quality in relation to health.

1. Office for National Statistics. 2015 [cited $20^{\text {th }}$ September 2017] Available from: https://www.ons.gov.uk/peoplepopulationandcommunity/birthsdeathsandmarriages/lifeexpectancies/articles/howhaslifeexpectancychangedovertime/2015-09-09.

2. Waijers P, Feskens E. 2005 [cited 5th September 2017] Available from: https://www.rivm.nl/bibliotheek/rapporten/35001003.pdf.

3. NatCen Social Research, MRC Elsie Widdowson Laboratory, UCL Medical School. [data collection]. UK Data Service. [cited $8^{\text {th }}$ November 2017] Available from: http://doi.org/10.5255/UKDA-SN-6533-7

4. Public Health England. The EatWell guide. 2016 [20th October 2017]. Available from: https://assets.publishing.service.gov.uk/government/uploads/ system/uploads/attachment_data/file/551502/Eatwell_Guide_booklet.pdf. 